\title{
Programas eTwinning para la adquisición de competencias del siglo XXI. Buscando una categoría central
}

\section{Etwinning programs for the acquisition of 21 st century skills. Looking for a central category}

\author{
María Nieto Martín ${ }^{1}$, Felipe Gértrudix Barrio ${ }^{2}$ \\ ${ }^{1}$ Universidad de Castilla la Mancha. maria.nieto2@uclm.es \\ ${ }^{2}$ Universidad de Castilla la Mancha.felipe.gertrudix@uclm.es
}

Recibido: $12 / 2 / 2021$

Aceptado: $22 / 10 / 2021$

Copyright $($ C)

Facultad de CC. de la Educación y Deporte. Universidad de Vigo

Dirección de contacto: Felipe Gértrudix Barrio

Facultad de Educación. Toledo Universidad de Castilla la Mancha

Avda. Carlos III, s/n

45071 Toledo

\section{Resumen}

ETwinning es la mayor plataforma europea de colaboración entre centros educativos y docentes, que mediante las tecnologías de la información y la comunicación desarrolla proyectos educativos entre dos o más países miembros. Con eTwinning, además de la adquisición de conocimientos, se contribuye a la generación en el alumnado de una serie de competencias y destrezas necesarias en la ciudadanía del siglo XXI. Se presenta un estudio en el que se analiza la vinculación existente entre la implementación de los programas de tele colaboración eTwinning en el escenario educativo y la adquisición de competencias del siglo XXI. Sobre los presupuestos de la teoría fundamentada, se ha llevado a cabo una revisión bibliográfica de la literatura internacional seleccionada por su relevancia e impacto. Se han analizado 44 documentos escritos en habla inglesa. Se han estudiado de manera individualizada diferentes componentes de las tecnologías de la información y la comunicación, de las competencias del siglo XXI y de los programas eTwinning, configurando una red que describe las interrelaciones entre estas variables. Como conclusión se declara que la implementación de programas eTwinning contribuye positivamente a la generación de competencias del siglo XXI.

\section{Palabras clave}

Etwinning, Colaboración, TIC en Educación, Competencias del Siglo XXI

\begin{abstract}
Etwinning is the largest European collaboration platform between educational centers and teachers, which through information and communication technologies develops educational projects between two or more member countries. By using eTwinning, in addition to the acquisition of knowledge, it contributes to the generation in students of a series of competences and skills which are necessary in the citizenship of $21 \mathrm{st}$ century. A study is presented in which the link between the implementation of eTwinning tele-collaboration programs in the educational setting and the acquisition of 21 st century skills is analyzed. On the assumptions of grounded theory, a
\end{abstract}


bibliographic review of the international literature selected for its relevance and impact has been carried out. 44 documents have been analyzed, which were written in the English language. Different components of information and communication technologies, 21 st century skills and eTwinning programs have been individually broken down to finally configure a network that describes the interrelationships between these variables. As a conclusion, it is declared that the implementation of eTwinning programs contributes positively to the generation of 21 st century skills.

\section{Key Words}

Etwinning, Collaboration, ICT in Education, 21st Century Skills

\section{INTRODUCCIÓN}

El uso de las Tecnologías de la Información y la Comunicación (TIC) ha permeado en diversos ámbitos de la vida de las personas, entre ellos, en el campo educativo. En un marco de uso adecuado, las TIC ofrecen innumerables oportunidades.

Según Sunkel y Truco (2010), las TIC no deben considerarse como un fin en sí mismas, sino que deben constituir un medio para la consecución de unos objetivos de desarrollo pedagógico. Asimismo, Selwyn (2002) señala que lo verdaderamente relevante de la introducción de las TIC en educación es el cambio metodológico que emerge a través de su uso. Si la información se transmite de la misma manera, pero a través de la tecnología, se continuarán empleando las dinámicas del aula tradicional y la metodología será ineficaz (Turoff, 1995).

De acuerdo con la UNESCO (2003), las TIC son herramientas fundamentales en la generación de sociedades del conocimiento. La Comisión Europea (2012), considera que las TIC en educación son un elemento clave en la creación de una educación de calidad. En esta línea, la estrategia de crecimiento Europa 2030, reconoce que la innovación educativa y la modernización de la educación y la formación son necesarias para la trasformación de la Unión Europea en relación con una economía competitiva e inclusiva. Esto se vincula directamente con algunas de las iniciativas clave de la estrategia Europa 2030, como son la agenda de nuevas cualificaciones y empleo, juventud en movimiento, la agenda digital y la agenda de la unión por la innovación. La Comisión Europea (2007) señala la competencia digital y la competencia básica en ciencia y tecnología como unas de las competencias clave que se establecen el marco de referencia de la Unión Europea.

En la Decisión número 2318/2003/CE del Parlamento Europeo y del Consejo, de 5 de diciembre de 2003, que adoptó un programa plurianual (2004-2006) para la integración efectiva de las TIC en los sistemas de educación y formación en Europa (programa eLearning), se recoge la necesidad de la incorporación de las TIC en los sistemas de formación y educación europeos. Esto se materializa en cuatro líneas de actuación: (a) fomento de la alfabetización digital, (b) campus europeos virtuales, (c) hermanamiento electrónico de centros europeos y fomento de la formación del profesorado, y (d) acciones transversales y de seguimiento del aprendizaje electrónico.

De la tercera línea surge el nacimiento de los proyectos eTwinning: programas de hermanamiento electrónico entre al menos dos centros escolares europeos que comparten intereses mutuos. Para la creación y puesta en marcha de estos proyectos es necesario el uso de las TIC, lo que promueve el aprendizaje y práctica de competencias 
digitales y la conciencia de la existencia de un modelo multicultural de la sociedad europea.

Algunos estudios precedentes los encontramos en Rivas-Rebaque et al. (2015) quienes establecen las claves para el empoderamiento de la ciudadanía mediante las políticas TIC, el desarrollo de la competencia digital, el open data y la alfabetización digital. Kirschner y Stoyanov (2020) recogen que el alumnado ahora presente en las aulas habrá de encontrarse con un campo laboral diferente al actual, con la existencia de profesiones aún desconocidas. Por ello, se requiere una preparación para el desempeño de unas tareas inciertas y una reformulación de los sistemas educativos. Entre estos cambios se señalan la necesidad de una relación integrada entre escuelas y el ámbito laboral, una profesionalización del ejercicio de la enseñanza y un rediseño de las escuelas como ecosistemas dónde los alumnos puedan llevar a cabo aprendizajes significativos y su desarrollo personal. Asimismo, destacan la promoción de la metacognición y la reflexión, el desarrollo del pensamiento crítico, la transferencia del conocimiento, una alfabetización informacional y la colaboración.

La conceptualización de la ciudadanía ha sufrido un cambio con relación a los derechos y deberes requeridos en el mundo contemporáneo. En este contexto, en el proyecto de la OCDE llamado Definition and Selection of Competences (DeSeCo) se establecen las competencias necesarias para la educación a lo largo de la vida. Las competencias ciudadanas se materializan en un "saber hacer" aplicable a contextos académicos, sociales y profesionales.

La Partnership for 21st century skills, P21, establece un marco pedagógico internacional para la inclusión de las competencias del siglo XXI. Estas incluyen habilidades para la vida personal y profesional, competencias de aprendizaje e innovación, competencia en el manejo de la información, de los medios y de las TIC. Dentro de este marco se promociona también la introducción de las 4Cs (critical thinking, communication, collaboration y creativity).

En este sentido, Trilling y Fadell (2009) recogen los cambios con relación a los objetivos de los sistemas educativos de la sociedad del siglo XXI, respecto a los de épocas anteriores, haciendo hincapié en la relación al trabajo y a la sociedad, enmarcados dentro de una economía y producción global, el ejercicio y desarrollo de los talentos personales de los alumnos, el cumplimiento de responsabilidades cívicas y en la aplicación del conocimiento.

Sobre la cultura, se requiere un desarrollo de una identidad cultural propia, así como la incursión dentro de una ciudadanía global con respeto hacia la multiculturalidad.

Trilling y Fadell (2009) materializan estos objetivos de la sociedad del siglo XXI en las competencias del siglo XXI, que son:

- Pensamiento crítico y resolución de problemas.

- Estrategias de comunicación y colaboración.

- Desarrollo de la creatividad e innovación.

Teniendo en cuenta todos estos precedentes, ¿en qué medida las TIC y los programas eTwinning contribuyen a la generación de competencias del siglo XXI? 


\section{OBJETIVOS}

El estudio tiene como objetivo principal indagar sobre cómo interrelacionan las TIC en el ámbito educativo y, en su caso, eTwinning y su contribución al desarrollo de las competencias del siglo XXI. Para ello, se investiga sobre las relaciones que se establecen entre las tres variables de investigación:

1. TIC en educación.

2. Competencias ciudadanas del siglo XXI.

3. Programas eTwinning.

Con todo ello los objetivos específicos son:

- Conocer el estado de las investigaciones a nivel internacional sobre TIC en educación.

- Indagar sobre qué competencias son las necesarias en los ciudadanos del siglo XXI y, por consiguiente, en el alumnado.

- Vincular la adquisición de competencias del ciudadano del siglo XXI con la puesta en práctica de programas eTwinning en el ámbito educativo.

\section{METODOLOGÍA}

La metodología utilizada ha sido la revisión bibliográfica de la literatura, que es empleada para la identificación, evaluación e interpretación de un campo de investigación concreto durante un espacio de tiempo determinado (Ramírez y García, 2018).

El proceso de revisión que se siguió se hizo basándose en el planteamiento de Grijalva et al. (2019), que reconocen las siguientes etapas: planeación, selección, extracción y ejecución.

En la fase de planeación se identifica cuál es el problema y se determina la pregunta, además de realizarse una selección de las fuentes. La pregunta que se planteó es: ¿en qué medida las TIC y los programas eTwinning contribuyen a la generación de competencias del siglo XXI?

Para la búsqueda se utilizaron la base de datos Scopus y Web of Science y para la organización y selección de los datos se empleó la aplicación web Mendeley.

Para la etapa de selección, se ha realizado una búsqueda independiente de cada una de las variables objeto de estudio. La relación resultante entre documento y variable concluyó de la siguiente manera: 14 para TIC en educación, 16 para competencias del siglo XXI y 14 para eTwinning, resultando un total de 44 documentos.

Los criterios de búsqueda han sido:

1. Los términos de recuperación que se han empleado combinados con los operadores son:

- Para la variable TIC en educación las palabras clave: "ICT" AND "education".

- Para la variable competencias del siglo XXI las palabras clave: "21st century skills" OR "21 st century competences" AND “education”.

- Para la variable eTwinning se emplea como palabra clave "eTwinning".

2. Como años de publicación se filtró desde 2009 hasta 2020.

3. Para el área del conocimiento se seleccionó Ciencias Sociales.

4. Como lengua se eligió inglés. 
En la etapa de extracción se realizó el filtrado, la selección y la clasificación de los documentos. En el proceso de filtrado de las fuentes que han sido analizadas han sido seleccionados un total de 2.003 estudios para la variable TIC en educación, un total de 475 estudios para la variable Competencias del siglo XXI y para la variable eTwinning 52 estudios. Posteriormente, se eliminaron los documentos duplicados en ambas bases de datos. Seguidamente, se siguieron criterios de inclusión para que se ajustasen a las temáticas innovación en TIC, desarrollo y políticas TIC, resultados obtenidos tras la aplicación de las TIC, definición y clasificación de las competencias, proyectos desarrollados que desarrollan las competencias, resultados de eTwinning y percepciones del alumnado y profesorado sobre eTwinning.

La etapa de ejecución comenzó con una codificación abierta, en la que se identifican los conceptos, las propiedades y dimensiones (Strauss y Corbin, 2002). Tras el proceso de depuración se llevó a cabo una codificación axial.

\section{RESULTADOS}

\subsection{Sobre las TIC en educación}

La Unión Europea ha establecido los indicadores de referencia que recogen el progreso en función de estos indicadores y objetivos para identificar las fortalezas y debilidades dentro de este proceso y extraer información útil para la elaboración de políticas a nivel nacional y europeo (The Survey of Schools: ICT in education). Los indicadores de referencia en los que se centra son: (a) competencia digital del alumnado y actitudes hacia las TIC, (b) uso de las TIC dentro y fuera del aula por parte de los alumnos, (c) uso de las TIC por parte de los docentes dentro y fuera del aula, (d) actitudes de los docentes hacia el uso educativo de estas tecnologías, (e) infraestructuras de los centros educativos, (f) conectividad y acceso y (g) papel de los equipos directivos con respecto a las tecnologías y su uso pedagógico.

En relación al estudio llevado a cabo por Alonso-García et al. (2019) relativo a buenas prácticas con TIC en diferentes universidades españolas destacamos los siguientes campos:

1. Ciencias Sociales y Ciencias Jurídicas, principalmente los entornos virtuales de aprendizaje, la web, la radio, los podcasts y los dispositivos móviles.

2. Artes y Humanidades con un desarrollo menor, son aplicados los ambientes virtuales de aprendizaje y los dispositivos móviles, con diferencias en las tecnologías usadas (fórums, blogs y wikis).

3. Arquitectura e Ingeniería han desarrollado pocas buenas prácticas con TIC, aunque se emplean una variedad de tecnologías $(3 \mathrm{D}$, podcasts, video y entornos virtuales de aprendizaje).

4. Ciencias, solamente son usados los entornos virtuales de aprendizaje como tecnología.

Bocconi et al. (2013) propusieron cinco trayectorias en el marco de la innovación en el aprendizaje usando las TIC:

1. Naturaleza de la innovación (incremental, radical, disruptiva)

2. Fase de implementación (piloto, de escalada, integración)

3. Nivel de acceso (local, regional/nacional, transfronterizo) 
4. Área de impacto (proceso, servicio, organización)

5. Objetivo (actores únicos, múltiples actores, amplio rango de actores.

Este análisis puede contribuir en la elaboración de políticas de intervención en niveles micro, macro y meso, las cuales se caracterizan por la diversidad, incrementando el impacto de las innovaciones educativas en las que se usan las TIC.

En esta línea, existen iniciativas de aprendizaje en Europa usando las TIC siguiendo el modelo computacional 1:1, las cuales tienen un alto grado de impacto en la educación y la formación, aunque simplemente equipar a profesores y alumnos con dispositivos electrónicos no es suficiente para realizar cambios transformadores de la educación (Valiente, 2010).

Asimismo, Sangrá y González-Sanmamed (2010) señalan que los esfuerzos de las administraciones y los gobiernos están centrados en dotar a las escuelas con el equipamiento necesario. Sin embargo, sería útil un análisis profundo de los usos educativos de las TIC. Según estos autores existirían cuatro niveles de escuelas con relación al uso que hacen de las TIC y según la combinación de los factores infraestructura, uso e innovación. Estos son:

1. Nivel 1: escuelas con uso educativo de las TIC limitado.

2. Nivel 2: escuelas que tienen una sala de ordenadores bien equipada.

3. Nivel 3: existe una o más sala de ordenadores bien equipada.

4. Nivel 4: escuelas que han decidido hacer de las TIC su elemento de distinción en sus actividades educativas.

Según este estudio existe una correlación entre el nivel en el que se encuentren las escuelas y las actitudes del profesorado con relación a las TIC, probablemente por el uso sistemático y generalizado que de estas realizan.

En esta misma línea Spiteri y Chang Rundgren (2018) señalan que, además de la cultura de la escuela en relación al uso de las TIC, son factores importantes la confianza, las creencias y los sentimientos de eficacia respecto a su uso por parte del profesorado.

Existe una relación recíproca entre la cultura del centro y el profesor puesto que el conocimiento del profesor y sus actitudes y destrezas son influidos por el centro y, a su vez, influyen en el centro. Además, la presencia de líderes digitalmente competentes, un buen servicio técnico y apoyo al uso son necesarios para integrar la tecnología de forma eficaz dentro de un centro (Kim y Keller, 2011). Las investigaciones diferencian entre el conocimiento del currículo tradicional y el currículo con tecnología; este último es más complejo y variado y permite una innovación en el contenido presentado en la clase (Aesaert et al., 2013).

El empleo de la tecnología en el aula requiere el cumplimiento de diferentes roles por parte del profesor y la organización de actividades adecuándose a las necesidades de los estudiantes. Existe una distinción entre tecnología fija y móvil, como la pizarra digital y los iPad, donde la primera puede ser utilizada siguiendo metodologías tradicionales de enseñanza, pero la última, debido a su movilidad requiere de una reorganización de la clase y cambios en el rol docente y del alumno (Orlando y Attard, 2016).

Además, Anastasiades y Vitalaki (2011) encontraron que los profesores que integraban la tecnología en su práctica diaria tenían más fácil promover hábitos seguros de uso de Internet mediante la discusión del tema con los alumnos.

En este sentido, Cabero et al. (2019) señalan la reconceptualización que ha sufrido la ciudadanía gracias a la aportación de las TIC en su empoderamiento y participación. El 
ejercicio de esta e-ciudadanía crítica requiere no solo de una adecuada formación política y cultural sino también de la competencia digital en los ciudadanos.

Esta e-ciudadanía tiene una forma diferente de relacionarse gracias a la aportación de diversas tecnologías y entornos digitales tales como Internet, el big data, las herramientas de la web 2.0, las tecnologías móviles, la inteligencia artificial, la computación en nubes, el software libre o las redes sociales. Los usuarios se han transformado de ser visitantes de la red a residir en la red (Rifkin, 2014).

Debemos admitir, por tanto, un cambio de visión sobre las tecnologías y la evolución que han sufrido desde el inicio como TIC (Tecnologías de la Información y la Comunicación) a otros modelos más innovadores que las conciben como TAC (Tecnologías de Aprendizaje y Conocimiento) y como TEP (Tecnologías de Empoderamiento y Participación) (Cabero et al., 2019). Por ello, las tecnologías se percibirían como instrumentos para la cooperación, la colaboración, la participación y el empoderamiento de las personas. Se considera, también, además del carácter individual de las TIC, la dimensión social y colectiva que estas poseen.

Es por tanto necesario el fortalecimiento de espacios de interacción como wikis, blogs, redes sociales o comunidades virtuales, donde exista una comunicación enriquecedora y asimétrica que propicie la reflexión y la creación de conocimientos, que tenga como consecuencia una mejora del pensamiento crítico y la mejora de la sociedad.

Para la construcción de esta sociedad crítica se debe reducir al máximo la brecha digital. Una de las formas de conseguirlo es mediante la incentivación de la alfabetización digital en todos los sectores poblacionales, y no solo en el período de escolarización (Kilbride, 2016).

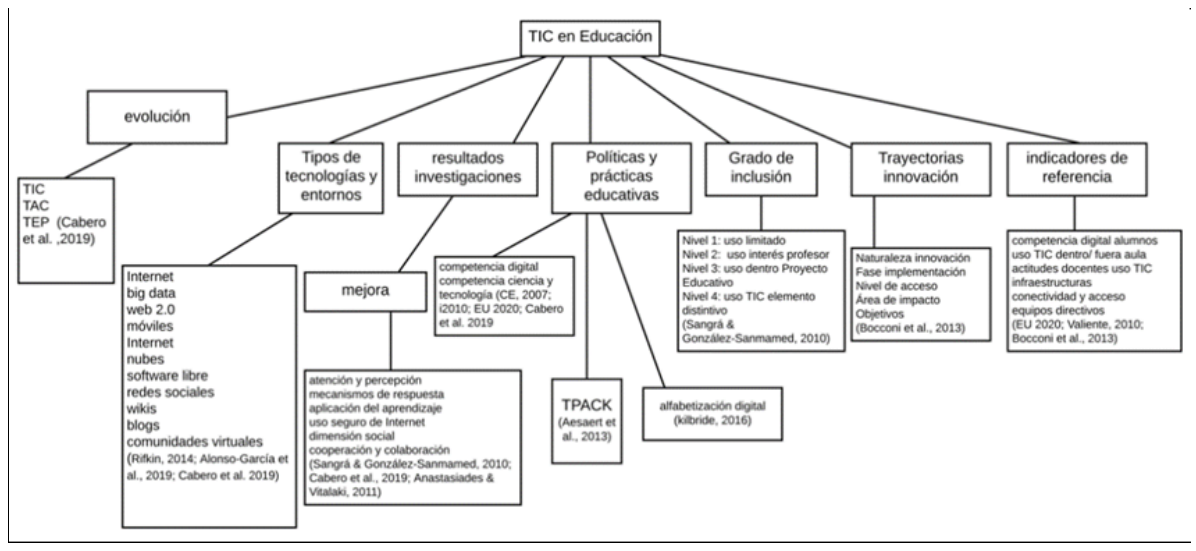

Figura 1. Mapa conceptual sobre TIC en educación

\subsection{Sobre las competencias del siglo XXI}

Las destrezas y competencias del siglo XXI están relacionadas con las necesidades de desarrollo social y de los modelos económicos emergentes, marcándose la diferencia con los del siglo pasado, los cuales se correspondían con un modelo más industrial de producción.

Los jóvenes están experimentando nuevas formas de socialización y de adquisición de capital social para los que el desarrollo de las TIC está contribuyendo (Ananiadou y Claro, 2009). Esto incluye procesos relacionados con la selección, adquisición, 
integración, análisis y el intercambio de información. La mayoría de estas competencias, si no todas, están apoyadas o mejoradas a través de las TIC.

Iniciativas sobre la enseñanza y evaluación de las competencias del siglo XXI de las que son parte integrante diferentes grupos - profesores, investigadores sobre educación, políticos, empleadores- recogen las competencias de este siglo para un desempeño adecuado en el trabajo, como ciudadanos y en el tiempo libre. Ejemplo de estas iniciativas es la Partnership for 21st skills, P21, (Asociación para las competencias del siglo XXI), que señala su importancia, no solo para investigadores y elaboradores de políticas, sino también para el sector privado.

Sin embargo, para Geisinger (2016), a pesar de que las competencias del siglo XXI son uno de los temas educativos más importantes, no se ha explorado en profundidad cómo medirlas.

Algunas voces críticas se han alzado ante tales propuestas, por ejemplo, el Common Core Group, que reclama un mayor énfasis hacia el contenido. Concluyen que, a pesar de considerar el concepto de competencia como extremadamente valioso, en algunos casos se configuran en función de las peticiones de los negocios y empresas y se sigue un enfoque economicista de la educación, debido a que su fin principal es preparar a los trabajadores para la producción económica o, incluso, para determinadas compañías.

Existen diferentes concepciones sobre cuáles son las competencias necesarias para el siglo XXI. Algunos autores consideran que las competencias para una sociedad moderna son el pensamiento crítico, la creatividad, la metacognición, la colaboración y la motivación (Gamage et al., 2014) y, además de estas, la perseverancia, el liderazgo y las habilidades sociales (Ghafar, 2020).

El propósito de estas competencias del siglo XXI es transformar a los individuos y hacer que puedan usar y aplicar el conocimiento en diferentes contextos. En esta línea, DiCicco (2016) vincula las competencias del siglo XXI a una educación ciudadana mundial en la que se fomente la justicia social y un desempeño activo de los ciudadanos en la sociedad.

Gupta (2020) añade a las competencias anteriores la comunicación, la alfabetización informacional, la alfabetización digital, la alfabetización sobre los medios de comunicación, la flexibilidad, la iniciativa y la productividad. Este autor señala que el aspecto común que tienen estas competencias es la adaptabilidad, que es la capacidad de adaptarse al cambio con nuevas ideas y metodologías y agrupa las competencias del siglo XXI en las siguientes categorías: (a) competencias de aprendizaje, (b) competencias literarias y (c) competencias para la vida.

Cada una de estas categorías se refiere a un objetivo de desarrollo específico, tal y como se describe en la Agenda 21, de las Naciones Unidas.

1. Las competencias de aprendizaje que ayudan a los estudiantes en sus procesos mentales, lo cual es necesario para adaptarse a un mundo moderno.

2. Los estudiantes adquieren conocimiento a través del uso de la tecnología.

3. Las competencias para la vida son las habilidades que un individuo posee en su vida personal, las cuales son reflejadas también en su vida profesional.

Otra forma de clasificar las diferentes destrezas y competencias es la propuesta por Ananiadou y Claro (2009), que distinguen entre aquellas que están más directamente relacionadas con las TIC y aquellas que no lo están. Estas son:

1. Competencias TIC funcionales

2. Competencias TIC educativas. 


\section{Competencias del siglo XXI.}

Dentro de las competencias del siglo XXI los autores reconocen tres dimensiones: información, comunicación e impacto ético y social.

Las competencias de la información implican la búsqueda, evaluación, selección, organización e interpretación de información. Además, la investigación sugiere que las TIC constituyen el marco adecuado para las habilidades de orden superior tales como el manejo, la información, el análisis crítico, la resolución de problemas y la creación de información.

Las competencias de la comunicación juegan un rol principal en la preparación de los estudiantes como miembros de una comunidad con voz propia y sentido de responsabilidad sobre los otros. Esta dimensión tiene dos subdimensiones: comunicación efectiva y colaboración e interacción virtual.

Las competencias relacionadas con el impacto ético y social están fundamentadas en la globalización, la multiculturalidad y el aumento del empleo de las $\mathrm{TIC}$, lo que implica retos éticos: (a) creatividad e innovación, (b) pensamiento crítico, (c) resolución de problemas y toma de decisiones, (d) aprender a aprender y metacognición, (e) alfabetización informacional, (f) alfabetización digital, (g) comunicación, (h) colaboración (trabajo en equipo), (i) ciudadanía global y local, (j) vida y carrera profesional, (k) responsabilidad personal y social -incluyendo conciencia y expresiones culturales-.

Para el desarrollo de estas competencias en el alumnado se requiere un curriculum fuerte y sostenible configurado para tal fin. De acuerdo con Van den Branden (2015), las necesidades de aprendizaje de los estudiantes del siglo XXI son hacer que el conocimiento funcione de forma práctica: en lugar de acumular saberes descontextualizados, este se debe emplear para dar respuesta de manera flexible a problemas complejos. Se debe propiciar dar solución a diferentes situaciones que pudieran producirse en un contexto real incentivando las resoluciones innovadoras y creativas.

Como parte de un proyecto internacional de investigación, "Assessment and Teaching of 21st century skills" (ATC21S), en el que se precisan las características del ciudadano competente del siglo XXI, se señalan como principios fundamentales la forma de pensar, las formas y herramientas para trabajar y las formas de vivir en el mundo.

La creatividad es la competencia más importante para dar respuesta a problemas contemporáneos (Newton y Newton, 2014). Bellanca y Brandt (2010) recalcan la necesidad de una toma de conciencia con relación a la inclusión de la creatividad. Sin embargo, aunque los currículos educativos resaltan la importancia de la creatividad, la innovación y el pensamiento crítico, los profesores en raras ocasiones lo ponen en práctica en forma de objetivos, resultados de aprendizaje o alentando a los alumnos a ser persistentes con una idea a pesar del fracaso.

En este orden de ideas, Romero et al. (2014) introdujeron el término de "cocreatividad", conjugándose los conceptos de colaboración y creatividad, dotando de una dimensión colectiva al concepto. Destacan la relevancia de la resolución de problemas de forma colaborativa como una de las competencias de mayor valor. Esta resolución de problemas requiere de una conjugación de destrezas, conocimiento y habilidades. Cuando esta solución se da de manera individual se emplean únicamente habilidades cognitivas o de comunicación. En cambio, cuando esta resolución de problemas se 
produce con la participación de otros individuos, además de las destrezas cognitivas, participan aspectos no cognitivos, tales como habilidades sociales y la gestión personal y de grupo. Dentro de estas estarían la autorregulación, la participación y la toma de perspectiva.

Según algunos investigadores existe una deficiencia en el desarrollo de las habilidades colaborativas del alumnado, por ejemplo, para Heinrichs (2016), que considera que el valor de esta competencia radica en su aplicación en contextos de aprendizajes auténticos y en el mundo real.

En lugar de enfatizar el éxito individual la educación del siglo XXI pretende el trabajo conjunto, la resolución pacífica de conflictos y el respeto hacia las decisiones del otro. Se debe enfocar la docencia hacia el descubrimiento de los propios talentos y limitaciones. Asimismo, se debe preparar a los estudiantes para el cambio mediante el ejercicio de la flexibilidad y preparación para situaciones inciertas.

Como se ha visto anteriormente, cada marco emplea una terminología y una categorización de las competencias distintas. A pesar de ser nombradas de diferente manera o estar enfocadas desde distinta perspectiva, existe una recurrencia en las competencias desde los diferentes marcos. Estas podrían categorizarse en cuatro grandes bloques: (a) competencias personales, (b) competencias sociales, (c) competencias de la información y del conocimiento, y (d) competencias en conocimientos digitales. De acuerdo con Voogt et al. (2012) se debe formar al profesorado basándose en las competencias del siglo XXI. Las TIC serían componentes indispensables en esta preparación de los docentes. En su estudio Valtonen et al. (2013) concluyen que una forma de formar a estos profesores es mediante TPACK (Technological Pedagogical Content Knowledge o Conocimiento Técnico Pedagógico del Contenido).

Además de lo anterior, dentro del marco de uso de las TIC, cabe mencionar que la educación actual genera una gran cantidad de información, pero los procesos educativos se llevan a cabo de manera opaca y no aportan suficiente información. Esto impide una mejora en la toma de decisiones y deriva en acciones de bajo impacto y resultados no lo suficientemente positivos. En este contexto, Learning Analytics, concebido como el proceso de visualización e interpretación de grandes cantidades de datos (big data) aplicado a la educación, ofrece la oportunidad a las diferentes partes intervinientes en el proceso educativo de estar mejor informados y, por ende, tomar mejores decisiones. (Ferguson, 2012). De esta forma, los centros educativos podrán llevar a cabo una formación más efectiva y personalizada recogiendo e interpretando la información adecuándose, ajustándose así a las demandas de una educación del siglo XXI. 


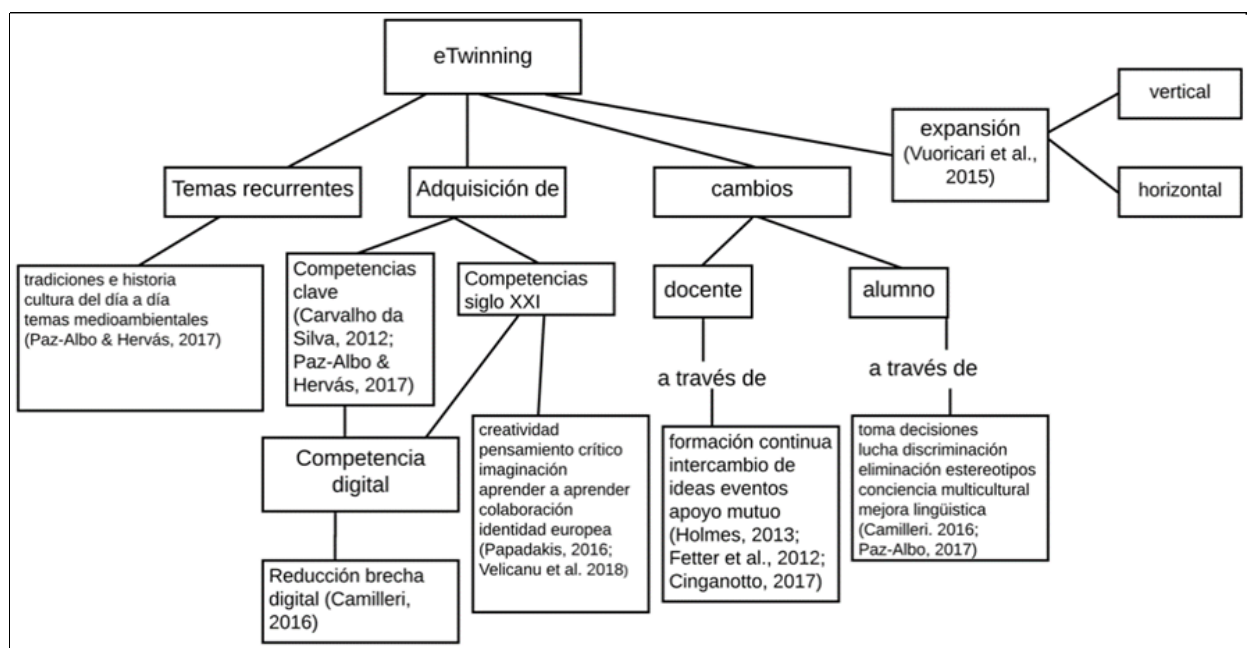

Figura 2. Mapa conceptual sobre Competencias del siglo XXI

\subsection{Sobre eTwinning}

La tecnología multimedia digital trae consigo oportunidades de aprendizaje mediante la conectividad, favoreciendo la telecolaboración entre docentes y alumnos en diferentes partes del mundo. La telecolaboración forma parte tanto de la educación formal como de la educación no formal a través de diferentes proyectos internacionales apoyados por instituciones públicas, organizaciones no gubernamentales $\mathrm{y}$ empresas privadas (Bozdağ, 2018).

Estas experiencias hacen que el alumno pertenezca a redes internacionales e interculturales a través de los medios digitales, lo cual es beneficioso en un mundo globalizado marcado por una importante diversidad cultural. De diferentes estudios en los que los estudiantes son encuestados sobre eTwinning se concluye que estos lo encuentran positivo y favorecedor sobre su aprendizaje (Paz-Albo y Hervás, 2017).

Los resultados del aprendizaje derivados de los programas eTwinning se vinculan con la adquisición de las Competencias Clave para el Aprendizaje Permanente elaboradas por la Comisión Europea (Carvalho da Silva, 2012; Paz-Albo y Hervás, 2017), además de con ciertas competencias del siglo XXI, tales como el desarrollo de la creatividad, el pensamiento crítico, la imaginación y la competencia de aprender a aprender (Velicanu et al., 2013).

Estos intercambios conllevan una serie de beneficios, como son: superar límites personales, conocer a personas con diferentes contextos culturales, estilos de vida, ideas, aprender con y de esas personas (Bozdag, 2018) y favorecer el intercambio de información y opiniones entre el alumnado y la cooperación entre las escuelas de Europa, contribuyendo a la formación de una identidad europea (Papadakis, 2016).

Además, Gajek (2018) encuentra las siguientes ventajas: despiertan la curiosidad intelectual entre el alumnado, mejoran las actitudes frente al aprendizaje, se desarrollan estrategias de resolución de problemas e incrementan la motivación.

Paz-Albo y Hervás (2017) llevaron a cabo el análisis de 1.800 proyectos eTwinning desarrollados en diferentes países europeos. Los resultados fueron:

1. Los estudiantes se dan cuenta de su pertenencia a una sociedad multicultural, mejorando sus conocimientos lingüísticos y culturales y, a su vez, identificando las señas de identidad cultural (Camilleri, 2016), así como muestran una apertura hacia 
la pluralidad de diferentes contextos sociales y culturales (Carvalho da Silva, 2012).

2. Respecto al contenido, estos presentan una serie de categorías que son recurrentes: cultura del día a día, tradiciones e historia, temas medioambientales, asignaturas específicas, cooperación multilingue y multicultural, temas que no se incluyen en el curriculum.

3. En referencia a los modelos de trabajo, estos suelen ser cooperativos y colaborativos. El proceso de enseñanza-aprendizaje sufre una remodelación y se dirige el foco hacia un curriculum orientado hacia el estudiante (Carvalho da Silva, 2012).

4. En cuanto a la mediación, esta se produce a nivel lingüístico y cultural en textos tanto orales como escritos. Los alumnos traducen textos de su lengua a una lengua extranjera, principalmente, inglés. Los profesores y estudiantes practican su competencia mediadora y competencia pragmática y los estudiantes incrementan su nivel de confianza en relación a su uso de una lengua extranjera (Carvalho da Silva, 2012; Velicanu et al., 2013).

5. En relación al rol docente, este sufre una transformación al producirse una apertura del aula a los ojos de otros docentes. El profesor incrementa sus niveles de satisfacción, experiencia y autoconfianza mediante el intercambio de buenas prácticas educativas y se vuelve un facilitador que guía a los estudiantes en su adquisición del conocimiento (Camilleri, 2016). ETwining, como red colaborativa, cumple tres premisas claves para el impulso del Capital Social dentro de una red de aprendizaje: se establecen relaciones entre los docentes, sentido de pertenencia -las personas sienten que forman parte de una red y apoyo mutuo-, las personas emplean las relaciones que se han establecido (Fetter et al., 2012).

6. Un gran número de estudios demuestran que los programas eTwinning tienen gran efectividad entre los alumnos y profesores como una oportunidad de implementar prácticas pedagógicas efectivas y proyectos que promuevan la adquisición de competencias del siglo XXI (Papadakis, 2016)

7. El rol del alumno sufre un cambio mediante la implementación de los proyectos eTwinning. Los estudiantes tienen que enfrentarse a la toma de decisiones en relación a tareas, al contenido, así como desarrollar competencias de colaboración y comunicación. Se promueve la ciudadanía y se lucha contra la discriminación y la generación de estereotipos (Camilleri, 2016; Paz-Albo y Hervás, 2017).

Algunas investigaciones a este respecto, como la de Cinganotto (2017) ahondan en los resultados producidos en el alumnado tras la implementación de programas eTwinning. Por su parte, Pinto y Escudeiro (2017), mediante la investigación-acción recogen las competencias desarrolladas en los estudiantes de once países, los cuales desarrollaron un juego usando el programa Scratch, en el contexto colaborativo de la plataforma eTwinning.

Para algunos alumnos que carecen de ordenador en su casa la participación en estos programas de telecolaboración es la única oportunidad para adquirir competencias tecnológicas, interculturales y lingüísticas. De esta forma, eTwinning puede contribuir a aminorar la brecha digital existente en el alumnado. Mediante la implementación de estos programas las competencias digitales en los estudiantes se ven mejoradas de una forma motivadora 
Para llevar a cabo este tipo de proyectos se necesita una competencia docente especial en la que se combinan lo tecnológico, lo pedagógico y el contenido. El énfasis debe ponerse en lo pedagógico y la tecnología debe ser un medio para tal fin (Camilleri, 2016).

En esta línea, Gouseti (2013) resalta que los proyectos eTwinning no deben ser los nuevos contextos en los que se introduzcan prácticas antiguas tales como copiar y pegar o trabajar individualmente, y en el que las plataformas online se empleen como meros repositorios.

En este orden de ideas, Gozalo et al. (2009) destacan el valor que poseen los programas eTwinning en el uso de las TIC como herramientas pedagógicas. Así la escuela será un medio para socializar, no solo dentro de la propia aula, sino en contextos europeos, lo cual redundará en beneficio de diferentes aspectos de la personalidad de los alumnos.

Papadakis (2016) vincula los programas eTwinning con el desarrollo profesional docente al favorecer la interacción de profesionales de diferentes disciplinas, lo que deriva en una mejora de conocimientos, personal, competencias y destrezas (Vuorikari et al., 2015), y de ideas y prácticas docentes (Velicanu et al., 2013). Además, el proceso de enseñanza-aprendizaje se volverá más enriquecedor al incluirse elementos curriculares de diferentes países, y no simplemente los nacionales. Adicionalmente, Holmes (2013) analizó la formación continua profesional docente mediante un evento eTwinning (eTwinning Learning Event) que se llevó a cabo de manera online. Estas comunidades aportan al docente un aprendizaje personalizado y auténtico en el que se produce un intercambio informal de buenas prácticas. Los aprendizajes extraídos son aplicables al contexto de la práctica diaria del docente.

En este sentido, Cinganotto (2017) estudió la participación docente en otro evento eTwinning, esta vez sobre CLIL (Content and Language Integrated Learning) o AICLE (Aprendizaje Integrado de Contenidos y Lenguas Extranjeras). Se extrajo que los profesores participaron de una manera activa, exponiendo sus impresiones de manera voluntaria en el blog y en el foro. De igual manera, se concluyó que los profesores tuvieron una mejora de sus competencias lingüísticas, digitales, transversales, sociales y metodológicas. 


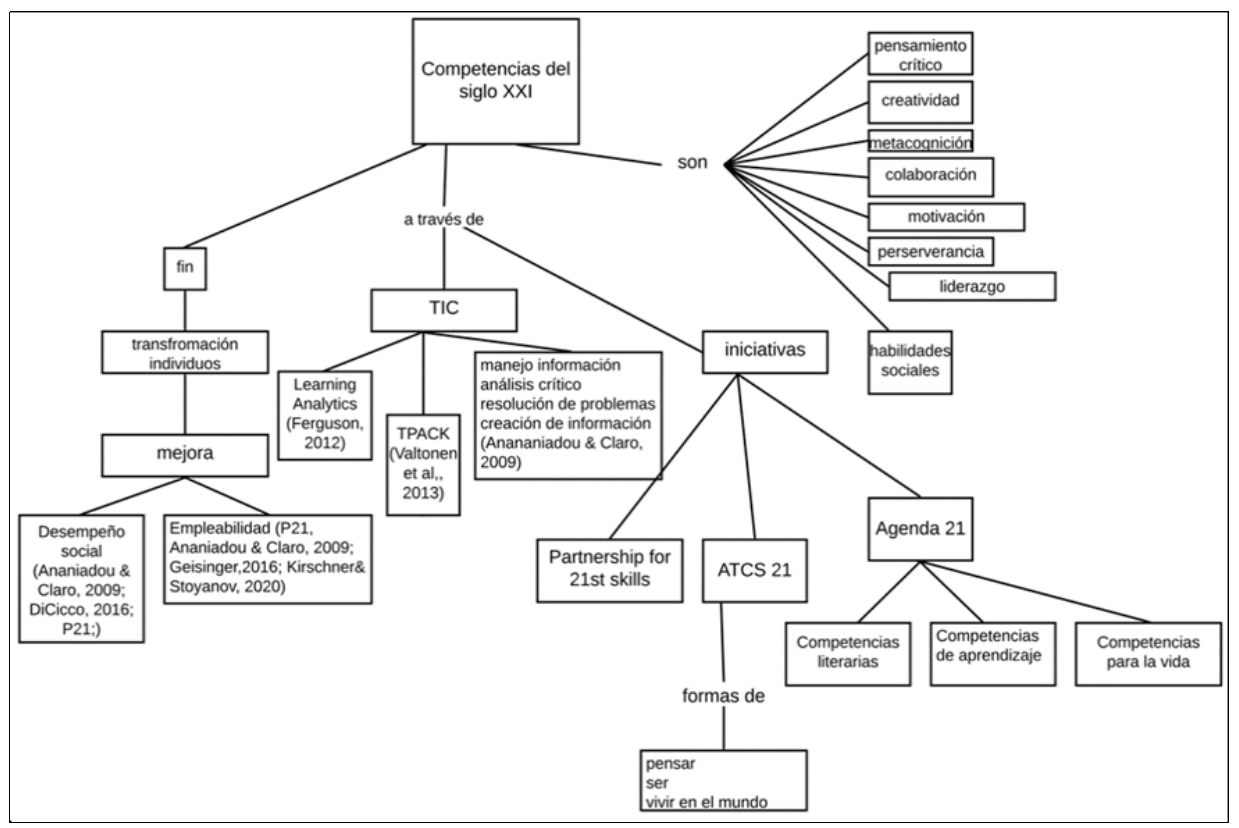

Figura 3. Mapa conceptual sobre eTwinning

De acuerdo con Vuorikari et al. (2015) con la implementación de eTwinning se produce un crecimiento tanto horizontal como vertical. El crecimiento horizontal se da cuando nuevos profesores de colegios que no estaban previamente registrados muestran interés en integrar esta red colaborativa. Es en este contexto donde los propios docentes trabajan hacia la mejora de los procesos educativos y los resultados. Estos sufren una transformación de sus creencias y principios pedagógicos, una reformulación de las normas que rigen las interacciones sociales, tanto con otros profesores como con los alumnos, lo que genera unos cambios profundos de las escuelas. El crecimiento vertical se da cuando dentro del colegio se producen oportunidades para que los docentes se desarrollen profesionalmente. La difusión de la innovación se realiza en parte gracias a los eTwinners invisibles, los cuales participan en el proyecto pero no aparecen registrados en la plataforma.

\subsection{Hacia una categoría central}

Mediante el análisis cualitativo de los documentos de cada una de las categorías se pueden concluir los siguientes resultados:

En general, existe una recurrencia entre el contenido presente en los documentos de las diferentes variables. Se han conectado los códigos provenientes de los documentos para establecer un sistema de relaciones.

Con esto, podemos establecer una relación entre las categorías para concluir que: la implementación de programas eTwinning contribuye a la generación de competencias del siglo XXI.

El siguiente diagrama muestra las relaciones entre las diferentes subcategorías, categorías y categoría central. 


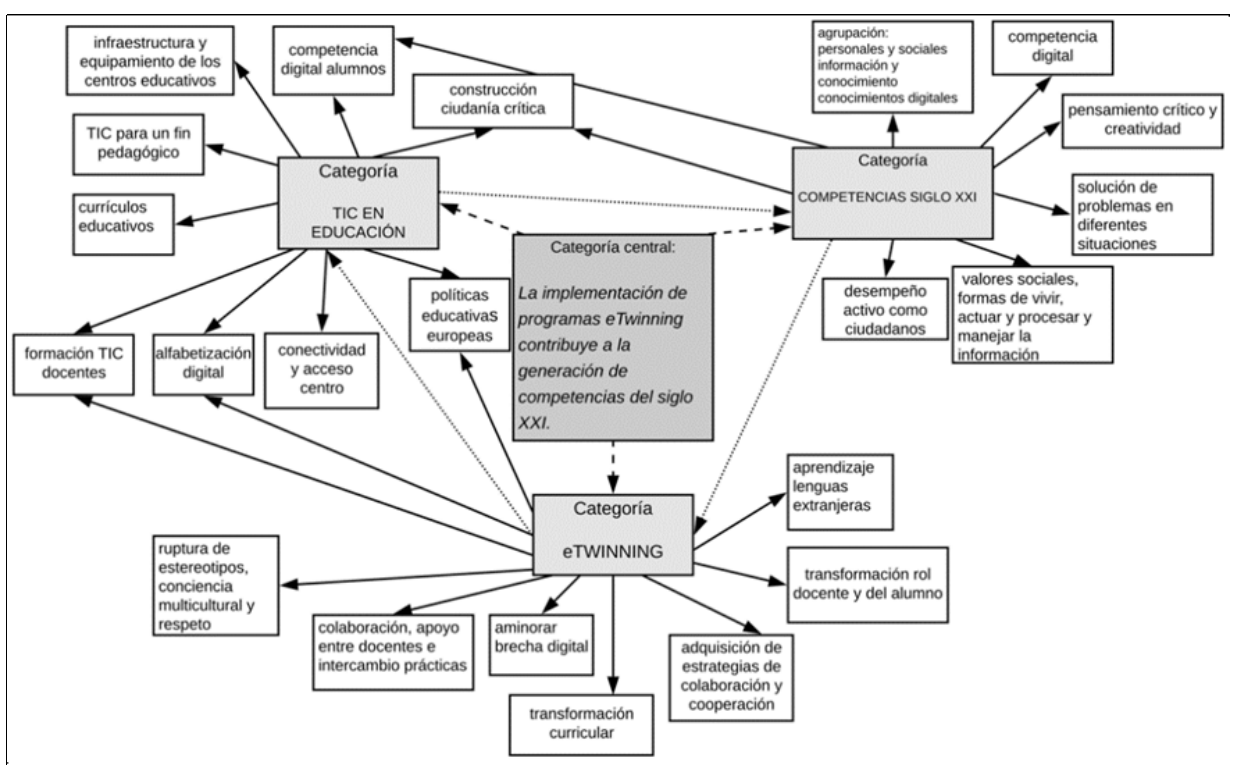

Figura 4. Categoría central y relación entre variables

\section{CONCLUSIONES Y DISCUSIÓN}

Los resultados obtenidos confirman el objeto de la investigación, al afirmarse que mediante la implementación de los programas eTwinning se fomenta el desarrollo de las competencias del siglo XXI, donde las TIC juegan un rol determinante en el desarrollo de este tipo de competencias (Ananiadou y Claro, 2009).

A partir del análisis de los datos extraídos podemos declarar las siguientes inferencias:

Las TIC en el ámbito educativo constituyen el medio para la consecución de determinados fines pedagógicos.

La literatura a este respecto hace hincapié en la importancia de mantener el foco pedagógico cuando se empleen las TIC, como señalan, por ejemplo, Turoff (1995), Selwyn (2002) o Sunkel y Trucco (2010). En este sentido Valiente (2010) defiende el modelo 1:1 hacia un enfoque pedagógico, en el que se vire la mirada desde los dispositivos digitales e infraestructuras hacia los alumnos y el proceso pedagógico. Desde diferentes organismos europeos se remarca la importancia del uso de las TIC en educación haciendo referencia al desarrollo de la competencia digital y mejora de las actitudes en el alumnado. Sin embargo, para Sangrá y González-Sanmamed (2010) los esfuerzos de las administraciones y gobiernos están puestos en equipar y dotar a los centros de herramientas tecnológicas, cuando sería preciso un análisis en profundidad de los usos que se están haciendo de las TIC en educación para una futura reformulación.

El grado de inclusión de las TIC está determinado por diversos factores.

Puede decidirse en función de factores tales como el grado de equipamiento y conectividad, la formación y actitudes del profesorado con respecto a su uso y la presencia en el proyecto de centro.

Existe mayor brecha digital en función del grupo social.

Existe una brecha digital de determinados grupos sociales con respecto al uso de las TIC en el entorno del hogar y, desde los centros escolares, en la medida de lo posible, se debe contribuir a aminorarla. En este sentido, Kilbride (2016) propone que se debe 
propiciar la formación digital, no solo en la etapa escolar, sino a lo largo de toda la vida y en diferentes sectores de la población. Camilleri (2016) otorga a los programas eTwinning valor como un factor que contribuye en la disminución de la brecha digital al ayudar al alumnado en su desarrollo de la competencia digital.

Un uso adecuado de las TIC aporta beneficios educativos.

Las TIC, dentro de un marco de uso adecuado, presentan beneficios extraídos de los diferentes documentos analizados como son: mejora en los mecanismos de respuesta y aplicación del conocimiento, incremento de la motivación y atención en el alumnado (Sangrá y González-Sanmamed, 2010).

Diversidad en la nomenclatura de las competencias del siglo XXI.

Las competencias del siglo XXI atienden a diferentes clasificaciones dependiendo del autor, algunas responden a distintas nomenclaturas. No obstante, pueden agruparse en competencias personales y sociales, competencias sobre el tratamiento de la información y el conocimiento y competencias digitales.

Competencias personales y de empoderamiento ciudadano vs competencias para el interés de la empresa.

Las competencias del siglo XXI permiten al ciudadano del siglo XXI alcanzar su potencial tanto como persona como en el ámbito laboral (P21; Geisinger, 2016; Kirschner y Stoyanov, 2020) siendo las competencias de mayor relevancia: el pensamiento crítico, la creatividad, la resolución de problemas, la colaboración y la comunicación. En contra de este modelo surgen algunas posiciones como el Common Core Group, que concluye que la determinación de estas competencias del siglo XXI se hace basándose en determinados intereses de las empresas, que persiguen crear empleados en función de sus necesidades.

El programa eTwinning aporta beneficios tanto a estudiantes como docentes en especial en estrategias de colaboración y cooperación

Los programas eTwinning contribuyen a la generación en el alumnado de competencias clave y de las competencias del siglo XXI (Carvalho da Silva, 2012; PazAlbo y Hervás, 2017; Velicanu et al., 2013). El programa eTwinning, en general, reporta beneficios tanto a nivel docente como del alumnado. Con respecto a los profesores, estos desarrollan estrategias de colaboración y cooperación con otros docentes, incorporándose buenas prácticas educativas a la acción profesional. Esta red de hermanamiento electrónico ofrece apoyo mutuo entre los docentes. Con respecto al alumnado, estos mejoran sus estrategias de cooperación e incorporan conocimientos multiculturales y lingüísticos a su bagaje. Esto favorece la ruptura de estereotipos y el respeto hacia las diferencias (Carvalho da Silva, 2012; Paz-Albo y Hervás, 2017).

\section{BIBLIOGRAFÍA}

Aesaert, K., Vanderlinde, R., Tondeur, J. y van Braak, J. (2013). The content of educational technology curricula: A cross-curricular state of the art. Educational Technology Research and Development, 61(1), 131-151. http://doi.org/f4mbhd

Alonso-García, S., Aznar-Díaz, I., Cáceres-Reche, M.P., Trujillo-Torres, J.M. y RomeroRodríguez, J.M. (2019). Systematic Review of Good Teaching Practices with ICT in Spanish Higher Education Trends and Challenges for Sustainability. Sustainability, 11(24). http://doi.org/dw7q 
Ananiadou, K. y Claro, M. (2009). 21st century skills and competences for new millennium learners in OECD countries. OECD Education Working Papers, 41(33). http://doi.org/fmp848

Anastasiades, P.S. y Vitalaki, E. (2011). Promoting Internet safety in Greek primary schools: The teacher's role. Journal of Educational Technology \& Society, 14(2), 71-80.

Bellanca, J. y Brandt, R. (2010). 21st century skills: rethinking how students learn. Solution Tree Press.

Bocconi, S., Kampylis, P. y Punie, Y. (2013). Framing ICT-enabled Innovation for Learning: The case of one-to-one learning initiatives in Europe. European Journal of Education, 48(1), 113-130. http://doi.org/gdz6bk

Bozdağ, Ç. (2018). Intercultural learning in schools through telecollaboration? A critical case study of eTwinning between Turkey and Germany. International Communication Gazette, 80(7), 677-694. http://doi.org/gfm6c8

Cabero, J., Torres-Barzabal, L. y Hermosilla-Rodríguez, J.M. (2019). Las TIC y la creación de una ciudadanía crítica e-digital. Education in the Knowledge Society, 20, 1-10. http://doi.org/dw7t

Camilleri, R.A. (2016). Global education and intercultural awareness in eTwinning. Cogent Education, 3(1). http://doi.org/c4zs

Carvalho da Silva, M. da P. (2012). "Learning from one another" eTwinning project: A model of an intercultural approach to using ICT in foreign language teaching. Handbook of Research on Didactic Strategies and Technologies for Education: Incorporating Advancements, 1, 170-181. http://doi.org/dw7x

Cinganotto, L. (2017). Experiential learning for teacher training: A case example on language, content and technologies in a learning event by eTwinning. Journal of E-Learning and Knowledge Society, 13(1), 91-111. http://doi.org/dw7w

Comisión Europea (2007). Competencias clave para el aprendizaje permanente. Un marco de referencia europeo. Comunidades Europeas: Oficina de Comunicaciones Oficiales.

Comisión Europea (2012). Developing Key Competences at School in Europe. Challenges and Opportunities for Policy. https://bit.ly/2oidzAR

DiCicco, M.C. (2016). Global citizenship education within a context of accountability and 21 st century skills: The case of olympus high school. Education Policy Analysis Archives, 24, 126. http://doi.org/dw7z

European Commission (2010). Europe 2020: a strategy for smart, sustainable and inclusive growth (COM [2010] 2020).

Ferguson, R. (2012). Learning analytics: drivers, development and challenges. International Journal of Technology Enhanced Learning, 4(6), 304-317.

Fetter, S., Berlanga, A.J., Sloep, P., Van Der Vegt, W., Rajagopal, K. y Brouns, F. (2012). Using peer-support to connect learning network participants to each other: an interdisciplinary approach. International Journal of Learning Technology, 7(4), 378-399.

Gajek, E. (2018). Curriculum integration in distance learning at primary and secondary educational levels on the example of etwinning projects. Education Sciences, 8(1), 1-15. http://doi.org/c4zv

Gamage, D., Perera, I. y Fernando, S. (2014). Improving eLearning to meet challenges in 21st century. 2014 14th International Conference on Advances in ICT for Emerging Regions, ICTer 2014- Conference Proceedings, 256. http://doi.org/dw72

Geisinger, K.F. (2016). 21st century skills: What are they and how do we assess them? Applied Measurement in Education, 29(4), 245-249

Ghafar, A., (2020). Convergence between 21st Century Skills and Entrepreneurship Education in Higher Education Institutes. International Journal of Higher Education, 9(1), 218-229. http://doi.org/dw73 
Gouseti, A. (2013). 'Old wine in even newer bottles': the uneasy relationship between web 2.0 technologies and European school collaboration. European Journal of Education, 48(4). 570-585. https://doi.org/10.1111/ejed.12051

Gozalo, L., Medina, C.J., Muñoz, A., Ortiz, C., Prestel, C., Rojas, D. y Sayalero, B. (2009). Nativos digitales, ciudadanos europeos: la acción eTwinning como ejemplo de la educación en colaboración a través de la red. Revista Icono 14(12), 123-141. https://doi.org/10.7195/ri14

Grijalva, P., Cornejo, G., Gómez, R., Real, K. y Fernández, A. (2019). Herramientas colaborativas para revisiones sistemáticas. Revista Espacios, 40(45), 9. Recuperado de: https://bit.ly/2AsNLbm

Gupta, M. (2020). 21st Century Skills and Sustainability. International Journal of Advanced Science and Technology, 28(16), 507-513.

Heinrichs, C.R. (2016). Exploring the influence of 21st century skills in a dual language program: A case study. (Doctoral dissertation). California State Polytechnic University, Pomona.

Holmes, B. (2013). School Teachers' Continuous Professional Development in an Online Learning Community: Lessons from a case study of an eTwinning Learning Event. European Journal of Education, 48(1), 97-12. http://doi.org/gd6ft8

Kilbride, J. (2016). Literacy and the digital divide. English in Aotearoa, 89, 33.

Kim, C.M. y Keller, J.M. (2011). Towards technology integration: The impact of motivational and volitional email messages. Educational Technology Research and Development, 59(1), 91-111. http://doi.org/b63jdh

Kirschner, P.A. y Stoyanov, S. (2020). Educating Youth for Nonexistent/Not Yet Existing Professions. Educational Policy, 34(3). http://doi.org/dw74

Leadbeater, C. y Wong, A. (2010) Learning from the Extremes. ICT in education (ICT4E) initiatives. New Media and Society. http://doi.org/dw75

Newton, L.D. y Newton, D.P. (2014). Creativity in 21st-century education. Prospects, 44(4), 575589. http://doi.org/dw8j

Orlando, J. y Attard, C. (2016). Digital natives come of age: the reality of today's early career teachers using mobile devices to teach mathematics. Mathematics Education Research Journal, 28(1), 107-121. http://doi.org/dw79

Papadakis, S. (2016). Creativity and innovation in European education. 10 years eTwinning. Past, present and the future. International Journal of Technology Enhanced Learning, 1(1), 1. http://doi.org/dw76

Paz-Albo, J. y Hervás, A. (2017). The Etwinning Experience: Beyond School Classrooms. ICERI2017 Proceedings, 1, 8.848-8.851. http://doi.org/c4zq

Pinto, A. y Escudeiro, P. (2017). The promotion of the century learning skills through the development of games using scratch. Iberian Conference on Information Systems and Technologies, CISTI, 10-15. http://doi.org/dw77

Ramírez, M.S. y García, F.J. (2018). Co-creación e innovación abierta: Revisión sistemática de literatura. Comunicar, 54, 9-18. http://doi.org/gctr7m

Rifkin, J. (2014). La Sociedad de coste marginal cero. Paidós.

Rivas-Rebaque, B., Gértrudix-Barrio, F. y De Cisneros de Britto, J.C. (2015). Análisis acerca de las claves en las Políticas Educativas para el Empoderamiento Ciudadano. Edutec, 53. http://doi.org/dw78

Romero, M., Usart, M. y Ott, M. (2014). Can serious games contribute to developing and sustaining 21st century skills? Games and Culture, 10(2), 148-177. http://doi.org/gcx5pw

Sangra, A. y Gonzalez-Sanmamed, M. (2010). The role of information and communication technologies in improving teaching and learning processes in primary and secondary schools. ALT-J: Research in Learning Technology, 18(3), 207-220. http://doi.org/dp7nx9

Selwyn, N. (2002). Telling tales on technology: Qualitative studies of technology and education. Ashgate. 
Spiteri, M. y Chang Rundgren, S. (2018). Literature review on the factors affecting primary teachers' use of digital technology. Technology, Knowledge and Learning, 25(1), 115-128. http://doi.org/dw8b

Strauss, A. y Corbin, J. (2002). Bases de la investigación cualitativa: Técnicas y procedimientos para desarrollar la teoría fundamentada. U. de Antioquia, Ed.

Sunkel, G. y Trucco, D. (2010). TIC para la educación en América Latina. Riesgos y oportunidades. Serie Políticas Sociales, 167, 1-7.

Trilling, B. y Fadel, C. (2009). 21st century skills: Learning for life in our times. JosseyBass/Wiley.

Turoff, M. (1995). Designing a virtual classroom. International Journal of Educational Telecommunications, 1(2), 245-262.

UNESCO. (2003). Communiqué of the ministerial roundtable on 'Towards Knowledge Societies'. UNESCO.

Valiente, O. (2010). 1-1 in education: current practices, international comparative research evidence and policy implications. OECD working papers, 44 (Paris, OECD Publishing).

Valtonen, T., Kontkanen, S., Dillon, P., Kukkonen, J. y Väisänen, P. (2014). Upper Secondary and Vocational Level Teachers at Social Software. Education and Information Technologies, 19(4), 763-779. Recuperado de:

https://www.learntechlib.org/p/159189/

Van den Branden, K. (2015). Sustainable education: exploiting students' energy for learning as a renewable resource. Sustainability, 7(5), 5.471-5.487.

http://doi.org/dw8c

Velicanu, A., Lungu, I., Diaconita, V. y Nisioiu, C. (2013). Cloud E-learning. The 9 th International Scientific Conference eLearning and software for Education, 380-386.

Voogt, J., Fisser, P., Roblin, N., Tondeur, J. y Braakt, J. (2012). Technological pedagogical content knowledge. A review of the literature. Journal of Computer Assisted Learning, 29(2), 109-121. http://doi.org/xhq

Vuorikari, R., Kampylis, P., Scimeca, S. y Punie, Y. (2015). Scaling up teacher networks across and within European schools: the case of eTwinning. En C. Looi, C. y L. Teh (eds.), Scaling Educational Innovations, (pp. 227-254). Springer.

http://dx.doi.org/10.1007/978-981-287-537-2

Wastiau, P., Blamire, R., Kearney, C., Quittre, V., Van de Gaer, E. y Monseur, C. (2013). The use of ICT in education: A survey of schools in Europe. European Journal of Education, 48(1), 11-27. http://doi.org/gdz6bg 